\title{
UNIVERSAL PARALLEL COMPOSITION LAWS AND THEIR REPRESENTATIONS
}

\author{
C. T. $\mathrm{NG}^{*}$
}

\section{Introduction.}

In the analysis of algorithms for a given machine, measured values of a number of algorithm-machine characteristics are frequently considered. These measures may be used to describe indirectly the structures of or between the algorithm and the machine. They may influence the design of a system.

Apart from the question of what characteristics are to be measured, how to measure them is of much concern. For complicated algorithms, accurate evaluation of a measure is by itself an enormous task and could become intolerable. On the other hand, the use of subjective or prejudice estimated measures may fail to reflect the true characteristics and bias our original goal. The compromise between the desired simplicity in deriving at the measure and the accuracy of the measure in reflecting a complexity may not be in hand always.

We shall consider measures that can be derived under simple laws. Measures that can be modulated under universal composition laws are of much interest. Their explicit representations will be deduced.

\section{Preliminaries and notations.}

We consider algorithms which are represented as programs written in a certain language. We further assume that the programs are well structured and are representable as finite encoded trees. Measures of program parameters are to be made via their encoded trees. Rooted trees with at least one node other than the root are to be considered. For convenience, the term "node" refers to nodes other than the root. Each node of an encoded tree is encoded by two parameters as follows.

Let $L=\{A, B, C, \ldots\}$ be a given set. For example, $L$ can be conceived as a set of primitive operations a machine accommodates, or as a set of built-in macro operations together with the micro operations.

* Research supported by the N.R.C. of Canada under grant no. A8212.

Received November 17, 1975; in revised form September 21, 1976. 
Let $\mathscr{L}=\{\mathscr{A}, \mathscr{B}, \mathscr{C}, \ldots\}$ be a set of encoded trees under evaluation. Each node of a tree admits a code in $L$ and a branching factor in the closed real interval $[0,1]$. The branching factors of nodes having the same parent constitute a complete probability distribution. We shall denote by $\Gamma_{n}$ the set

$$
\left\{\left(p_{1}, p_{2}, \ldots, p_{n}\right) \mid p_{i} \in[0,1], \sum p_{i}=1\right\}
$$

of all complete probability distributions of length $n, n=1,2,3, \ldots$ The following example is a declaration of an encoded tree $\mathscr{A}$.

$$
\begin{array}{cc}
\text { ExAMPLE 2.1. } \mathscr{A}: \begin{array}{c}
1 \\
\end{array}\left(p_{1}, A\right), \\
2 a\left(q_{1}, B\right), \\
2 b\left(q_{2}, C\right), \\
3 a\left(r_{1}, A\right), \\
3 b\left(r_{2}, C\right), \\
1 b\left(p_{2}, D\right) \\
1 c\left(p_{3}, A\right), \\
2 c(1, E) ;
\end{array}
$$

whose graph is shown in Figure 1.

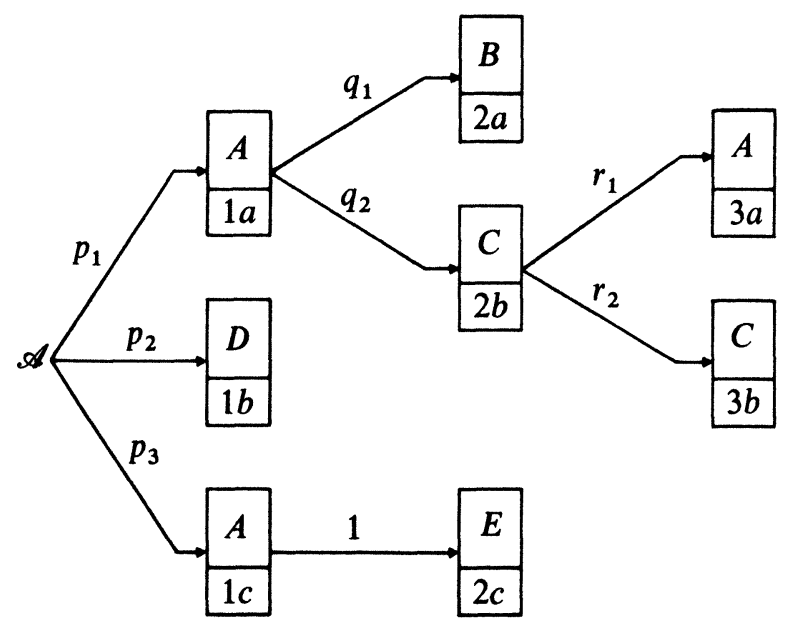

Figure 1.

The letter $\mathscr{A}$ qualifies the encoded tree, and the letters $a, b, c, \ldots$, qualify the nodes with the same offset number. Reference to a node in a tree can be made by giving the name of the tree, the offset number of the node, and the name of the node.

Parallel composition is an essential operation on $\mathscr{L}$ and will be described as follows. 
Let $\mathscr{A}_{1}, \mathscr{A}_{2}, \ldots, \mathscr{A}_{n}$ be encoded trees, and let $\left(p_{1}, p_{2}, \ldots, p_{n}\right) \in \Gamma_{n}$ be given. The parallel concatenation of $\left(\mathscr{A}_{i}\right)_{i}$ under $\left(p_{i}\right)_{i}$ is obtained by multiplying the branching factors of nodes with offset number one in $\mathscr{A}_{i}$ by $p_{i}$ and then formally joining them at the roots. Each node originally in $\mathscr{A}_{i}$ should be qualified by $\mathscr{A}_{i}$ if necessary. The resulting tree will be denoted by $\sum_{i}\left(p_{i}, \mathscr{A}_{i}\right)$ or $\left(p_{1}, \mathscr{A}_{1}\right)+\left(p_{2}, \mathscr{A}_{2}\right)+\ldots+\left(p_{n}, \mathscr{A}_{n}\right)$. This operation is symmetric in the $\left(p_{i}, \mathscr{A}_{i}\right)$ 's and can be multi-staged.

For example, if

ExAMPle 2.2. $\mathscr{A}_{1}: 1 a(1, A), 2 a\left(q_{1}, B\right), 2 b\left(q_{2}, C\right), 3 a\left(r_{1}, A\right), 3 b\left(r_{2}, C\right)$;

$\mathscr{A}_{2}: 1 b(1, D)$;

$\mathscr{A}_{3}: 1 c(1, A), 2 c(1, E)$;

then the tree $\mathscr{A}$ in Example 2.1 is equal to $\sum_{i}\left(p_{i}, \mathscr{A}_{i}\right)$, and is also equal to $\left(p_{1}, \mathscr{A}\right)+\left(p_{2}+p_{3},\left(p_{2} /\left(p_{2}+p_{3}\right), \mathscr{A}_{2}\right)+\left(p_{3} /\left(p_{2}+p_{3}\right), \mathscr{A}_{3}\right)\right)$ provided that $p_{2}+p_{3}$ $>0$.

We assume that $\mathscr{L}$ is closed under this operation, or else we consider subclasses of $\mathscr{L}$ which are closed under this operation.

\section{Postulates on the measures.}

(3.1) The measure of a program parameter can be derived via the encoded tree representing the program. Thus we may consider the measure $\mu$ as a mapping of $\mathscr{L}$ into the reals $\mathrm{R}$. We confine ourselves to consider real-valued measures.

(3.2) The measure $\mu$ is invariant under isomorphisms of encoded trees.

Thus the names of the nodes of an encoded tree are irrelevant in the evaluation of $\mu$. For the subsequent discussions we shall declare encoded trees without naming the nodes.

(3.3) Parallel compositivity: There exists a parallel composition law PAR such that

$$
\mu\left(\sum_{i=1}^{n}\left(p_{i}, \mathscr{A}_{i}\right)\right)=\operatorname{PAR}\left(\left(p_{i}, \mu \mathscr{A}_{i}\right)_{i=1}^{n}\right) .
$$

Intuitively speaking, the measure of a composed program $\sum_{i}\left(p_{i}, \mathscr{A}_{i}\right)$ can be composed by the measures $\mu \mathscr{A}_{i}$ of the individual modules $\mathscr{A}_{i}$ and their corresponding weights under some law PAR.

(3.4) Parallel locality: There exist functions $\alpha$ and $\Delta$ such that

$$
\mu\left(\left(p_{1}, \mathscr{A}_{1}\right)+\left(p_{2}, \mathscr{A}_{2}^{\prime}\right)\right)=\alpha^{-1}\left(\alpha \mu\left(\left(p_{1}, \mathscr{A}_{1}\right)+\left(p_{2}, \mathscr{A}_{2}\right)\right)+\Delta\left(\mathscr{A}_{2}, \mathscr{A}_{2}^{\prime}, p_{2}\right)\right) .
$$

Intuitively speaking, if a module $\mathscr{A}_{2}$ of a composed program $\left(p_{1}, \mathscr{A}_{1}\right)$ 
$+\left(p_{2}, \mathscr{A}_{2}\right)$ is, for some reason, modified or rewritten as $\mathscr{A}_{2}^{\prime}$, then it is possible to derive at the measure $\mu\left(\left(p_{1}, \mathscr{A}_{1}\right)+\left(p_{2}, \mathscr{A}_{2}^{\prime}\right)\right)$ via the original measure $\mu\left(\left(p_{1}, \mathscr{A}_{1}\right)+\left(p_{2}, \mathscr{A}_{2}\right)\right)$ and an additive correction term $\Delta\left(\mathscr{A}_{2}, \mathscr{A}_{2}^{\prime}, p_{2}\right)$ which depends locally on the original module, the modified module and the weight of that module in the composed program, after an appropriate change $\alpha$ of the measuring scale.

Remark 3.1. It does not matter whether (3.3) is to hold for all $n=2,3, \ldots$, or just for $n=2$, as parallel concatenation can be multi-staged.

In the coming section, some properties of the function PAR will be explored. It is natural to expect that PAR satisfies some properties in order that the measure $\mu$ composed under PAR fulfils the above postulates and is consistent with the structure of $\mathscr{L}$.

\section{Some properties of the parallel composition law PAR.}

Let $\mu: \mathscr{L} \rightarrow \mathrm{R}$ be a mapping satisfying all the proposed postulates (3.1) to (3.4), and let PAR, $\alpha$ and $\Delta$ be such asserted functions. (The existence of these functions is guaranteed by the postulates.)

In view of the compositivity of $\mu$, the correction term $\Delta\left(\mathscr{A}_{2}, \mathscr{A}_{2}^{\prime}, p_{2}\right)$ depends on $\mathscr{A}_{2}$ and $\mathscr{A}_{2}^{\prime}$ via their measures. Thus there exists a function $\delta$ such that

$$
\Delta\left(\mathscr{A}_{2}, \mathscr{A}_{2}^{\prime}, p_{2}\right)=\delta\left(\mu \mathscr{A}_{2}, \mu \mathscr{A}_{2}^{\prime}, p_{2}\right),
$$

and the function $\delta$ will replace $\Delta$ for the remaining discussions. No further consideration of $\Delta$ is necessary.

The postulated properties of $\mu$ are invariant under the various choices of measuring scales. To be precise, if $\beta$ is an injective mapping of $S:=\mu(\mathscr{L})$ onto a subset $T$ of $R$, then the induced measure $\bar{\mu}:=\beta \circ \mu$ is again a measure on $\mathscr{L}$ satisfying the asserted postulates under the functions $\overline{\mathrm{PAR}}, \bar{\delta}, \bar{\alpha}$ where

$$
\left\{\begin{array}{l}
\overline{\operatorname{PAR}}\left(\left(p_{i}, \bar{\mu} \mathscr{A}_{i}\right)_{i}\right):=\beta\left(\operatorname{PAR}\left(\left(p_{i}, \beta^{-1}\left(\bar{\mu} \mathscr{A}_{i}\right)\right)_{i}\right)\right) \\
\bar{\delta}\left(\bar{\mu} \mathscr{A}_{2}, \bar{\mu} \mathscr{A}_{2}^{\prime}, p_{2}\right):=\delta\left(\beta^{-1}\left(\bar{\mu} \mathscr{A}_{2}\right), \beta^{-1}\left(\bar{\mu} \mathscr{A}_{2}^{\prime}\right), p_{2}\right) \\
\bar{\alpha}(t):=\alpha\left(\beta^{-1}(t)\right), \quad t \in T .
\end{array}\right.
$$

Two measures are said to be isomorphic if they differ by a scaling function $\beta$. This relation partitions the measures into isomorphic classes. It is sufficient to explore measures that are "canonical".

A measure $\mu$ satisfying the asserted postulates with $\alpha$ equal to the identity map will be called canonical. Thus canonical measures are those which admit an additive correcting term. Every measure $\mu$ is isomorphic to a canonical measure (by taking $\beta=\alpha$, say). It is therefore sufficient to explore all parallel 
composition laws that give rise to canonical measures. All measures are supposed to satisfy all postulates in Section 3 from now on without explicit declaration.

LEMMA 4.1. If $\mu$ is canonical then there exist functions $f$ and $g$ representing PAR through

$$
\operatorname{PAR}\left(\left(p_{i}, u_{i}\right)_{i=1}^{n}\right)=f\left(p_{1}, p_{2}, \ldots, p_{n}\right)+\sum_{i=1}^{n} g\left(p_{i}, u_{i}\right)
$$

for all $\left(p_{i}\right) \in \Gamma_{n}, n=2,3, \ldots$, and for all $u_{i} \in \mu(\mathscr{L})$.

Proof. Let $\mathscr{A}_{0} \in \mathscr{L}$ be arbitrary but fixed and let $a_{0}=\mu\left(\mathscr{A}_{0}\right)$. Let $\mathscr{A}_{i} \in \mathscr{L}$ and $u_{i}=\mu\left(\mathscr{A}_{i}\right), i=1,2, \ldots, n$. We can evaluate PAR through $\delta$ as follows.

$$
\begin{aligned}
\operatorname{PAR} & \left(p_{1}, u_{1} ; p_{2}, u_{2} ; \ldots ; p_{n}, u_{n}\right)=\operatorname{PAR}\left(\left(p_{i}, \mu\left(\mathscr{A}_{i}\right)\right)_{i}\right) \\
= & \mu\left(\left(p_{1}, \mathscr{A}_{1}\right)+\left(p_{2}, \mathscr{A}_{2}\right)+\ldots+\left(p_{n}, \mathscr{A}_{n}\right)\right) \\
= & \mu\left(\left(p_{1}+p_{2}+\ldots+p_{n-1},\left(q_{1}, \mathscr{A}_{1}\right)+\left(q_{2}, \mathscr{A}_{2}\right)+\ldots+\left(q_{n-1}, \mathscr{A}_{n-1}\right)\right)+\right. \\
& \left.+\left(p_{n}, \mathscr{A}_{n}\right)\right)
\end{aligned}
$$

where $\left(q_{1}, \ldots, q_{n-1}\right) \in \Gamma_{n-1}$ is a distribution such that

$$
p_{j}=\left(p_{1}+p_{2}+\ldots+p_{n-1}\right) q_{j}, \quad j=1,2, \ldots, n-1 .
$$

Accordingly,

$$
\begin{aligned}
& \operatorname{PAR}\left(p_{1}, u_{1} ; p_{2}, u_{2} ; \ldots ; p_{n}, u_{n}\right) \\
&=\mu\left(\left(p_{1}+p_{2}+\ldots+p_{n-1},\left(q_{1}, \mathscr{A}_{1}\right)+\left(q_{2}, \mathscr{A}_{2}\right)+\ldots+\left(q_{n-1}, \mathscr{A}_{n-1}\right)\right)+\right. \\
&\left.+\left(p_{n}, \mathscr{A}_{0}\right)\right)+\delta\left(\mu\left(\mathscr{A}_{0}\right), \mu\left(\mathscr{A}_{n}\right), p_{n}\right) \\
&= \mu\left(\left(p_{1}, \mathscr{A}_{1}\right)+\left(p_{2}, \mathscr{A}_{2}\right)+\ldots+\left(p_{n-1}, \mathscr{A}_{n-1}\right)+\left(p_{n}, \mathscr{A}_{0}\right)\right)+\delta\left(a_{0}, \mu\left(\mathscr{A}_{n}\right), p_{n}\right) \\
&= \operatorname{PAR}\left(p_{1}, u_{1} ; p_{2}, u_{2} ; \ldots ; p_{n-1}, u_{n-1} ; p_{n}, a_{0}\right)+\delta\left(a_{0}, u_{n}, p_{n}\right) .
\end{aligned}
$$

This can be extended by the symmetry in parallel concatenation to

$\operatorname{PAR}\left(p_{1}, u_{1} ; p_{2}, u_{2} ; \ldots ; p_{n}, u_{n}\right)$

$$
=\operatorname{PAR}\left(p_{1}, a_{0} ; p_{2}, a_{0} ; \ldots ; p_{n}, a_{0}\right)+\sum_{i=1}^{n} \delta\left(a_{0}, u_{i}, p_{i}\right) .
$$

This proves our assertion with $g(p, u):=\delta\left(a_{0}, u, p\right)$ and

$$
f\left(p_{1}, p_{2}, \ldots, p_{n}\right):=\operatorname{PAR}\left(p_{1}, a_{0} ; p_{2}, a_{0} ; \ldots ; p_{n}, a_{0}\right) .
$$

LEMMA 4.2. The function $f$ in Lemma 4.1 satisfies the following properties: 
(i) Symmetry: $f\left(p_{1}, p_{2}, \ldots, p_{n}\right)$ is invariant under permutations of the $p_{i}$ 's, all $n=2,3, \ldots$

(ii) Branching: $f\left(p_{1}, p_{2}, \ldots, p_{n}\right)-f\left(p_{1}, p_{2}, \ldots, p_{n-2}, p_{n-1}+p_{n}\right)$ is a function of $p_{n-1}$ and $p_{n}$, i.e. the difference does not depend on $p_{1}, \ldots, p_{n-2}$ nor on $n$ as long as $\sum_{i=1}^{n-2} p_{i}$ remains constant.

Proof. (i) The symmetry of $f$ follows directly from that of $\operatorname{PAR}\left(\left(p_{i}, u_{i}\right)_{i=1}^{n}\right)$ and of $\sum_{i=1}^{n} g\left(p_{i}, u_{i}\right)$ in the $\left(p_{i}, u_{i}\right)$ 's.

(ii) For $\left(p_{1}, p_{2}, \ldots, p_{n}\right) \in \Gamma_{n}$, let $\left(q_{1}, q_{2}\right) \in \Gamma_{2}$ be such that $p_{n-1}=\left(p_{n-1}+p_{n}\right) q_{1}$, $p_{n}=\left(p_{n-1}+p_{n}\right) q_{2}$ and consider the equality

$$
\begin{aligned}
& \left(p_{1}, \mathscr{A}_{1}\right)+\left(p_{2}, \mathscr{A}_{2}\right)+\ldots+\left(p_{n-2}, \mathscr{A}_{n-2}\right)+ \\
& \quad+\left(p_{n-1}+p_{n},\left(q_{1}, \mathscr{A}_{n-1}\right)+\left(q_{2}, \mathscr{A}_{n}\right)\right)=\sum_{i=1}^{n}\left(p_{i}, \mathscr{A}_{i}\right) .
\end{aligned}
$$

The measure of the left hand side according to its suggested multi-stage composition is given by

$$
\begin{aligned}
f\left(p_{1}, p_{2}, \ldots, p_{n-2}, p_{n-1}+p_{n}\right)+\sum_{i=1}^{n-2} g\left(p_{i}, u_{i}\right)+ \\
+g\left(p_{n-1}+p_{n}, f\left(q_{1}, q_{2}\right)+g\left(q_{1}, u_{n-1}\right)+g\left(q_{2}, u_{n}\right)\right),
\end{aligned}
$$

while the right hand side has measure given by

$$
f\left(p_{1}, \ldots, p_{n}\right)+\sum_{i=1}^{n} g\left(p_{i}, u_{i}\right),
$$

where $u_{i}=\mu\left(\mathscr{A}_{i}\right)$. Thus we get

$$
\begin{aligned}
& f\left(p_{1}, p_{2}, \ldots, p_{n}\right)-f\left(p_{1}, p_{2}, \ldots, p_{n-2}, p_{n-1}+p_{n}\right) \\
= & g\left(p_{n-1}+p_{n}, f\left(q_{1}, q_{2}\right)+g\left(q_{1}, u_{n-1}\right)+g\left(q_{2}, u_{n}\right)\right)-g\left(p_{n-1}, u_{n-1}\right)-g\left(p_{n}, u_{n}\right) .
\end{aligned}
$$

Since the right hand side is a function of $p_{n-1}$ and $p_{n}$ while fixing $u_{n-1}, u_{n}$ and choosing $\left(q_{1}, q_{2}\right) \in \Gamma_{2}$ properly, this proves the lemma.

\section{Lemma 4.3. A function $f$ satisfies}

(i) Symmetry: $f\left(p_{1}, \ldots, p_{n}\right)$ is invariant under permutations of the $p_{i}$ 's, all $n=2,3, \ldots$, and

(ii) Branching: There exists a two-place function $\tilde{\Delta}$ such that

$$
\begin{gathered}
f\left(p_{1}, p_{2}, \ldots, p_{n}\right)-f\left(p_{1}, p_{2}, \ldots, p_{n-2}, p_{n-1}+p_{n}\right)=\tilde{\Delta}\left(p_{n-1}, p_{n}\right) \\
\quad \text { for all }\left(p_{1}, p_{2}, \ldots, p_{n}\right) \in \Gamma_{n} \text { and for all } n=3,4, \ldots,
\end{gathered}
$$

if, and only if, there exists a one-place function $h:[0,1] \rightarrow R$ such that $f$ is represented as: 


$$
f\left(p_{1}, p_{2}, \ldots, p_{n}\right)=\sum_{i=1}^{n} h\left(p_{i}\right)
$$

for all $\left(p_{1}, p_{2}, \ldots, p_{n}\right) \in \Gamma_{n}, n=2,3, \ldots$

Proof. Let $f$ be a function satisfying the symmetry and branching properties.

It follows immediately from the symmetry of $f$ that $\tilde{\Delta}$ is also symmetric, i.e.

$$
\tilde{\Delta}(x, y)=\tilde{\Delta}(y, x)
$$

for all $x, y \in[0,1]$ with $x+y \in[0,1]$. By using the symmetry and branching formula, we get

$$
\begin{aligned}
f(1-x-y-z, x, y, z) & =f(1-x-y-z, x, y+z)+\tilde{\Delta}(y, z) \\
& =f(1-x-y-z, x+y+z)+\tilde{\Delta}(x, y+z)+\tilde{\Delta}(y, z)
\end{aligned}
$$

and

$$
\begin{aligned}
f(1-x-y-z, x, y, z) & =f(1-x-y-z, x+y, z)+\tilde{\Delta}(x, y) \\
& =f(1-x-y-z, x+y+z)+\tilde{\Delta}(x+y, z)+\tilde{\Delta}(x, y) .
\end{aligned}
$$

By comparison we get

$$
\tilde{\Delta}(x, y)+\tilde{\Delta}(x+y, z)=\tilde{\Delta}(x, y+z)+\tilde{\Delta}(y, z)
$$

for all $x, y, z \in[0,1]$ with $x+y+z \in[0,1]$. By applying a result in $[6$, Theorem 2.1 and Remark 2.1], the function $\tilde{\Delta}$ can be represented by a one-place function $\tilde{h}:[0,1] \rightarrow R$ through

$$
\tilde{\Delta}(x, y)=\tilde{h}(x)+\tilde{h}(y)-\tilde{h}(x+y)
$$

for all $x, y \in[0,1]$ with $x+y \in[0,1]$. This explicit form of $\tilde{\Delta}$ enables us to calculate $f$ through $\tilde{h}$ as follows:

$$
\begin{aligned}
f\left(p_{1}, p_{2}, \ldots, p_{n}\right)= & f\left(p_{1}, p_{2}, \ldots, p_{n}, 0\right)-\tilde{\Delta}\left(p_{n}, 0\right) \\
= & -\tilde{h}(0)+f\left(p_{1}, p_{2}, \ldots, p_{n}, 0\right) \\
= & -\tilde{h}(0)+f\left(p_{1}+p_{2}, p_{3}, \ldots, p_{n}, 0\right)+\tilde{\Delta}\left(p_{1}, p_{2}\right) \\
= & -\tilde{h}(0)+f\left(p_{1}+p_{2}+p_{3}, p_{4}, \ldots, p_{n}, 0\right)+ \\
& +\tilde{\Delta}\left(p_{1}+p_{2}, p_{3}\right)+\tilde{\Delta}\left(p_{1}, p_{2}\right) \\
= & \ldots \\
= & -\tilde{h}(0)+f(1,0)+\sum_{i=1}^{n-1} \tilde{J}\left(\sum_{i=1}^{j} p_{i}, p_{j+\mathrm{r}}\right)
\end{aligned}
$$




$$
\begin{aligned}
& =-\tilde{h}(0)+f(1,0)+\sum_{i=1}^{n} \tilde{h}\left(p_{i}\right)-\tilde{h}(1) \\
& =-\tilde{h}(0)+f(1,0)-\tilde{h}(1)+\sum_{i=1}^{n} \tilde{h}\left(p_{i}\right)
\end{aligned}
$$

for all $\left(p_{1}, p_{2}, \ldots, p_{n}\right) \in \Gamma_{n}, n=2,3, \ldots$ If we define $h$ by $h(p):=\tilde{h}(p)+p f(1,0)$ $-p \tilde{h}(1)-p \tilde{h}(0)$ for all $p \in[0,1]$, we get the representation (4.3.1). The converse of Lemma 4.3 is obvious.

The above representation of $f$ enables us to strengthen Lemma 4.1 and obtain the following result by setting $k(p, u):=g(p, u)+h(p)$.

Proposition 4.4. If $\mu$ is canonical, then there exists a function $k$ representing PAR through

$$
\operatorname{PAR}\left(\left(p_{i}, u_{i}\right)_{i=1}^{n}\right)=\sum_{i=1}^{n} k\left(p_{i}, u_{i}\right),
$$

for all $\left(p_{i}\right) \in \Gamma_{n}, n=2,3, \ldots$, and for all $u_{i} \in \mu(\mathscr{L})$.

Thus, to determine PAR explicitly, it is sufficient to determine the two-place function $k$. The converse of Lemma 4.1 and, in particular, that of Proposition 4.4 are also obvious. We can now release the functions $f, g, h$ and $\delta$ as they are no longer needed.

The remaining condition on the composition law PAR such that the canonical measure $\mu$ is compatible with the structure of $\mathscr{L}$ is to follow. Let $\mathscr{A}_{i} \in \mathscr{L}, i=1,2,3$ and $p, q \in[0,1]$ be arbitrarily given. Consider the identity

$$
\left(1-p, \mathscr{A}_{1}\right)+\left(p,\left(1-q, \mathscr{A}_{2}\right)+\left(q, \mathscr{A}_{3}\right)\right)=\left(1-p, \mathscr{A}_{1}\right)+\left(p(1-q), \mathscr{A}_{2}\right)+\left(p q, \mathscr{A}_{3}\right)
$$

and their measures under PAR, we get

$$
\begin{aligned}
k\left(1-p, u_{1}\right)+k\left(p, k\left(1-q, u_{2}\right)+k\left(q, u_{3}\right)\right) & \\
= & k\left(1-p, u_{1}\right)+k\left(p(1-q), u_{2}\right)+k\left(p q, u_{3}\right)
\end{aligned}
$$

where $u_{i}=\mu \mathscr{A}_{i}$. After cancelling common terms we get

$$
k\left(p, k\left(1-q, u_{2}\right)+k\left(q, u_{3}\right)\right)=k\left(p(1-q), u_{2}\right)+k\left(p q, u_{3}\right)
$$

for all $p, q \in[0,1]$ and $u_{2}, u_{3} \in \mu(\mathscr{L})$. This observation leads to the following conclusion.

Theorem 4.5. (i) Each measure $\mu: \mathscr{L} \rightarrow \mathrm{R}$ satisfying the postulates (3.1) to (3.4) is isomorphic to a canonical measure satisfying again all the postulates (3.1) to (3.4) with a equal to the identity map on $\mu(\mathscr{L})$, 
(ii) Each such canonical measure admits a parallel composition law PAR having the following representation:

$$
\operatorname{PAR}\left(\left(p_{i}, u_{i}\right)_{i=1}^{n}\right)=\sum_{i=1}^{n} k\left(p_{i}, u_{i}\right)
$$

for all $\left(p_{i}\right) \in \Gamma_{n}, u_{i} \in \mu(\mathscr{L}), n=2,3, \ldots$, where $k$ is a function from $[0,1] \times \mu(\mathscr{L})$ into $R$ satisfying the equation

$$
k(p, k(1-q, u)+k(q, v))=k(p(1-q), u)+k(p q, v)
$$

for all $p, q \in[0,1]$ and all $u, v \in \mu(\mathscr{L})$.

Remark 4.6. Further exposure of PAR relies on our ability in solving the functional equation for $k$ in Theorem 4.5. The difficulty lies on the specific set $\mu(\mathscr{L})$ of acceptable values and that $\sum_{i=1}^{n} k\left(p_{i}, u_{i}\right)$ has to have values within $\mu(\mathscr{L})$. This problem is complex. The next section is along these lines.

\section{Regular canonical universal parallel composition laws PAR and their} representations.

Definition 5.1. Let $J$ be a non-degenerated interval of R. A regular canonical universal parallel composition law on $J$ is a mapping

$$
\text { PAR: } \bigcup_{n=2}^{\infty} \Gamma_{n} \times J^{n} \rightarrow \mathrm{R}
$$

representable by

$$
\operatorname{PAR}\left(p_{1}, p_{2}, \ldots, p_{n} ; u_{1}, u_{2}, \ldots, u_{n}\right)=\sum_{i=1}^{n} k\left(p_{i}, u_{i}\right),\left(p_{i}\right) \in \Gamma_{n}, u_{i} \in J,
$$

where $k:[0,1] \times J \rightarrow R$ is a continuous mapping satisfying the conditions

$$
k(1-q, u)+k(q, v) \in J,
$$

and

$$
\begin{gathered}
k(p, k(1-q, u)+k(q, v))=k(p(1-q), u)+k(p q, v) \\
\text { for all } p, q \in[0,1], u, v \in J
\end{gathered}
$$

Remark 5.2. We call it canonical as it is a law for canonical measures, universal as it acts on an interval $J$ which may host various measures, and regular as it is continuous. The fact that the PAR values will remain in $J$ is implicit, in view of (5.1.0) and (5.1.1). 
Remark 5.3. All functions refered to in this section are local to this section. We proceed to solve the functional equation (5.1.1) for continuous $k$. The following uniqueness theorem (see [5, Theorem 2.1]) will be applied immediately.

Lemma 5.4. Let $X, Y$ be intervals of $\mathrm{R}$ and $T: X \times Y \rightarrow \mathrm{R}$ be continuous in each variable. Let $f: X \rightarrow \mathrm{R}, g: Y \rightarrow \mathrm{R}, h: T(X \times Y) \rightarrow \mathrm{R}$ be functions and consider the functional equation

$$
f(x)+g(y)=h(T(x, y)) \quad \text { for all } x \in X, y \in Y .
$$

If $\left(f^{0}, g^{0}, h^{0}\right)$ is a particular solution with $f^{0}$ being continuous and non-constant and $g^{0}$ being non-constant. Then the general solution $(f, g, h)$ with a continuous $f$ is given by

$$
f=\alpha f^{0}+\beta_{1}, \quad g=\alpha g^{0}+\beta_{2}, \quad h=\alpha h^{0}+\beta_{1}+\beta_{2}
$$

where $\alpha, \beta_{1}, \beta_{2}$ are arbitrary real constants.

Proposition 5.5. Let $k:[0,1] \times J \rightarrow R$ be a continuous map satisfying (5.1.0) and (5.1.1). Suppose that for each $p \in[0,1]$, the map $u \rightarrow k(p, u)$ is non-constant on $J$. Then $k$ is given by

$$
k(p, u)=l(u)+B p-B,
$$

where $B$ is a constant, and $l: J \rightarrow R$ is a non-constant continuous map satisfying the constraints

(i) $l(J)+l(J)-B \cong J$

(The set $l(J)+l(J)-B=\{l(u)+l(v)-B \mid u, v \in J\}$ will actually be the set of values taken by PAR).

(ii) The restriction of $l$ to $l(J)+l(J)-B$ is the identity mapping.

The converse is also true.

Proof. For each fixed pair $p, q \in[0,1]$ in equation (5.1.1), we can apply Lemma 5.4 by taking $X=Y=J$ and

$$
\begin{aligned}
T(u, v) & :=k(1-q, u)+k(q, v) \\
f^{0}(u) & :=k(1-q, u) \\
g^{0}(v) & :=k(q, v) \\
h^{0} & :=\text { the identity map on } T(J \times J) \\
f(u) & :=k(p(1-q), u)
\end{aligned}
$$




$$
\begin{aligned}
& g(v):=k(p q, v) \\
& h(t):=k(p, t) \text { on } T(J \times J) .
\end{aligned}
$$

All hypotheses of Lemma 5.4 are satisfied and so there exist constant $\alpha(p, q)$, $\beta_{1}(p, q), \beta_{2}(p, q)$ such that

$$
\begin{array}{ll}
k(p(1-q), u) & =\alpha(p, q) k(1-q, u)+\beta_{1}(p, q) \\
k(p q, v) & =\alpha(p, q) k(q, v)+\beta_{2}(p, q) \\
k(p, t) & =\alpha(p, q) t+\beta_{1}(p, q)+\beta_{2}(p, q)
\end{array}
$$

for all $u, v \in J, t \in I_{q}:=\{k(1-q, u)+k(q, v) \mid u, v \in J\}$.

It follows from the last identity in (5.5.3) that for each fixed $p \in[0,1]$, the map $t \rightarrow k(p, t)$ is linear on each interval $I_{q}$. Further, $I_{q}$ is non-degenerated because the map $u \rightarrow k(p, u)$ is non-constant.

As the intervals $I_{q}$ are deforming continuously (loosely speaking) with respect to the parameter $q$, the linearity of the map $t \rightarrow k(p, t)$ can be extended to

$$
I_{[0,1]}:=\bigcup_{q \in[0,1]} I_{q} .
$$

Thus $\alpha(p, q)$ and $\beta_{1}(p, q)+\beta_{2}(p, q)$ are independent of $q$, and we can define unambiguously

$$
\begin{aligned}
& \alpha(p):=\alpha(p, q) \\
& \beta(p):=\beta_{1}(p, q)+\beta_{2}(p, q)
\end{aligned}
$$

and extend the third equation in (5.5.3) to

$$
k(p, t)=\alpha(p) t+\beta(p)
$$

for all $t \in I_{[0,1]}$ which is a subinterval of $J$. The continuity of $k$ implies the continuity of both $\alpha$ and $\beta$, since $I_{[0,1]}$ is non-degenerated. We now proceed to determine $\alpha$ and $\beta$ explicitly by using (5.5.4) and the original equations (5.1.0) and (5.1.1).

By confining $u, v$ in (5.1.1) to $I_{[0,1]}$ we get

$$
\begin{aligned}
\alpha(p) \alpha(1-q) u+\alpha(p) \alpha(q) v & +\alpha(p)[\beta(1-q)+\beta(q)]+\beta(p) \\
= & \alpha(p(1-q)) u+\alpha(p q) v+\beta(p(1-q))+\beta(p q)
\end{aligned}
$$

for all $u, v \in I_{[0,1]}$. This is equivalent to

$$
\left\{\begin{array}{l}
\alpha(p) \alpha(q)=\alpha(p q) \\
\alpha(p) \beta(1-q)+\alpha(p) \beta(q)+\beta(p)=\beta(p(1-q))+\beta(p q)
\end{array}\right.
$$

for all $p, q \in[0,1]$. Thus $\alpha$ is a continuous solution to the Cauchy's functional 
equation. Furthermore, $\alpha$ never vanishes on $[0,1]$. For otherwise there exists $p_{0} \in[0,1]$ such that $\alpha\left(p_{0}\right)=0$ and so $t \rightarrow k\left(p_{0}, t\right)$ is constant on $I_{[0,1]}$. This and (5.1.1) imply that $u \rightarrow k\left(p_{0}(1-q), u\right)$ is constant on $J$ for each $q \in[0,1]$, which is a contradiction to our hypotheses in this theorem. The solution for $\alpha$ is given by

$$
\alpha(p)=1 \text { for all } p \in[0,1] .
$$

The equation in $\beta$ reduces to

$$
\beta(1-q)+\beta(q)+\beta(p)=\beta(p(1-q))+\beta(p q)
$$

for all $p, q \in[0,1]$. By applying a result in [7] (Proposition 3, taking $F=\beta$, $B(p)=-\beta(p)-\beta(1-p)$ and $A \equiv 0)$, the continuous $\beta$ is given by

$$
\beta(p)=B p-B \quad \text { for all } p \in[0,1],
$$

where $B$ is a constant. With these explicit forms of $\alpha$ and $\beta$, and by setting $q=0$ in (5.1.1), we get

$$
k(1, u)+B p-B=k(p, u)
$$

for all $p \in[0,1], u \in J$. This is the asserted equation (5.5.1) where $l(u):=k(1, u)$. A direct verification shows that a function $k$ defined by (5.5.1) satisfies (5.1.0) and (5.1.1) if, and only if, the constraints (5.5.2) are satisfied. This completes our proof.

PROPOSITION 5.6. Let $k:[0,1] \times J \rightarrow \mathrm{R}$ be a continuous map satisfying (5.1.0) and (5.1.1). Suppose that for each $p \in] 0,1]$, the map $u \rightarrow k(p, u)$ is non-constant on $J$, and that the map $u \rightarrow k(0, u)$ is constant on $J$. Then $k$ is given either by

$$
k(p, u)=p^{a} l(u)+A p^{a}-A p
$$

with the convention $0^{a}:=0$

where $a, A$ are constant with $a>0$ and $a \neq 1, l: J \rightarrow R$ is a non-constant continuous map satisfying the constraints

$$
\text { (i) } \begin{aligned}
I_{[0,1]}:= & \left\{q^{a} l(u)+(1-q)^{a} l(v)+A\left[q^{a}+(1-q)^{a}-1\right] \mid\right. \\
& q \in[0,1], u, v \in J\} \cong J
\end{aligned}
$$

(ii) the restriction of $l$ to $I_{[0,1]}$ is the identity mapping,

or $b y$

$$
k(p, u)=p l(u)+C p \log _{2} p
$$

with the convention $0 \log _{2} 0:=0$,

where $C$ is a constant, $l: J \rightarrow R$ is a non-constant continuous map satisfying the constraints 
(i) $I_{[0,1]}:=\left\{q l(u)+(1-q) l(v)+C\left[q \log _{2} q+(1-q) \log _{2}(1-q)\right] \mid\right.$ $q \in[0,1], u, v \in J\} \subseteq J$

(ii) the restriction of $l$ to $I_{[0,1]}$ is the identity mapping.

The converse is also true.

Proof. The proof will be analogous to that of Proposition 5.5. Duplicated details will be omitted.

For each fixed $p \in[0,1], q \in] 0,1[$ in equation (5.1.1), we can apply Lemma 5.4 and so there exist constants $\alpha(p, q), \beta_{1}(p, q), \beta_{2}(p, q)$ such that

$$
k(p(1-q), u)=\alpha(p, q) k(1-q, u)+\beta_{1}(p, q)
$$

$$
\begin{array}{ll}
k(p q, v) & =\alpha(p, q) k(q, v)+\beta_{2}(p, q) \\
k(p, t) & =\alpha(p, q) t+\beta_{1}(p, q)+\beta_{2}(p, q)
\end{array}
$$

for all $u, v \in J, t \in I_{q}:=k(1-q, J)+k(q, J)$.

It follows from the last identity in (5.6.5) that for each fixed $p \in[0,1]$, the map $t \rightarrow k(p, t)$ is linear on each non-degenerated interval $I_{q}$ for all $\left.q \in\right] 0,1[$. As the intervals $I_{q}$ are deforming continuously with respect to the parameter $q$, the linearity of the map can be extended to

$$
I_{\mathrm{J}, 1 \mathrm{1}[}:=\bigcup_{q \in] 0,1[} I_{q}
$$

The continuity of $u \rightarrow k(p, u)$ allows us to conclude that the map is linear on $I_{[0,1]}$ which is contained in the topological closure of $I_{\mathrm{j} 0,1[}$. Thus, for each $p \in[0,1]$ there exist constant $\alpha(p), \beta(p)$ such that

$$
k(p, t)=\alpha(p) t+\beta(p)
$$

for all $t \in I_{[0,1]} \subseteq J$. The continuity of $k$ implies the continuity of $\alpha$ and $\beta$. We proceed to determine the explicit forms of $\alpha$ and $\beta$ by using (5.6.6) and the original equations (5.1.0) and (5.1.1).

By confining $u, v$ in (5.1.1) to $I_{[0,1]}$ using (5.6.6), and separating the free variables $u, v$, we get

$$
\left\{\begin{array}{l}
\alpha(p) \alpha(q)=\alpha(p q) \\
\alpha(p) \beta(1-q)+\alpha(p) \beta(q)+\beta(p)=\beta(p(1-q))+\beta(p q)
\end{array}\right.
$$

for all $p, q \in[0,1]$. Thus $\alpha$ is a continuous solution to Cauchy's functional equation. Furthermore, as we assumed that $u \rightarrow k(0, u)$ is constant on $J$ and thus constant on $I_{[0,1]}$, we have $\alpha(0)=0$. The fact that $\alpha(p) \neq 0$ for $p \neq 0$ follows from similar reasonings as those in the proof of Proposition 5.5. This leads to the explicit form of $\alpha$ 


$$
\alpha(p)=p^{a},
$$

with the convention $0^{a}:=0$,

for all $p \in[0,1]$, where $a>0$ is a constant.

The equation for $\beta$ in (5.6.7) is reduced to

$$
p^{a}[\beta(1-q)+\beta(q)]+\beta(p)=\beta(p(1-q))+\beta(p q)
$$

for all $p, q \in[0,1]$. The function $\beta$ is continuous and its explicit form will be solved as follows. There are two cases to be considered.

CASE 1. Suppose $a=1$ in (5.6.9).

In this case $\beta(p)-\beta(p(1-q))-\beta(p q)=-p[\beta(1-q)+\beta(q)]$ and thus $p \rightarrow \beta(p)-\beta(p(1-q))-\beta(q)$ is additive for each fixed $q \in[0,1]$. By applying a result in [7] (Proposition 3 with $F=\beta$ and $B=0$ ), it follows that $\beta$ is given by

$$
\beta(p)=C p \log _{2} p
$$

$$
\text { with the convention } 0 \log _{2} 0:=0 \text {, }
$$

where $C$ is a constant. From (5.6.6), (5.6.8) and (5.6.10) we get

$$
k(p, t)=p t+C p \log _{2} p
$$

for all $t \in I_{[0,1]} \leqq J$. By using this explicit form of $k$ on $[0,1] \times I_{[0,1]}$, confining $v$ to $I_{[0,1]}$ and setting $q=0$ in (5.1.1), we get

$$
p k(1, u)+C p \log _{2} p=k(p, u)
$$

for all $p \in[0,1]$ and $u \in J$. This is the asserted equation (5.6.3) where $l(u)$ : $=k(1, u)$ on $J$. A straightforward verification shows that the function $k$ given by $l$ through (5.6.3) satisfies the underlying equations (5.1.0) and (5.1.1) if, and only if, the constraints (5.6.4) are satisfied.

CASE 2. Suppose $a \neq 1$ in (5.6.9).

We replace $p$ in (5.6.9) by $(1-p)$ and add the resulting identity to (5.6.9) side by side so as to obtain

$$
\begin{aligned}
{\left[(1-p)^{a}+p^{a}\right][\beta(1-q)+\beta(q)]+\beta(1-p)+\beta(p) } \\
=\beta(p(1-q))+\beta(p q)+\beta((1-p)(1-q))+\beta((1-p) q) .
\end{aligned}
$$

The right hand side of the above identity is symmetric in $p$ and $q$ and therefore the left hand side is invariant if we interchange $p$ and $q$. Thus we have

$$
\begin{aligned}
{\left[(1-p)^{a}+p^{a}\right][\beta(1-q)+\beta(q)]+\beta(1-p)+\beta(p) } \\
=\left[(1-q)^{a}+q^{a}\right][\beta(1-p)+\beta(p)]+\beta(1-q)+\beta(q) .
\end{aligned}
$$


If we set $p=\frac{1}{2}$ we get immediately

$$
\beta(1-q)+\beta(q)=A\left[(1-q)^{a}+q^{a}-1\right]
$$

where $A:=2 \beta\left(\frac{1}{2}\right) /\left(2^{1-a}-1\right)$ is a constant. This explicit form of $\beta(1-q)+\beta(q)$ reduces (5.6.9) to

$$
p^{a} \cdot A\left[(1-q)^{a}+q^{a}-1\right]+\beta(p)=\beta(p(1-q))+\beta(p q)
$$

which is equivalent to

$$
\beta(p)-A p^{a}=\left[\beta(p(1-q))-A p^{a}(1-q)^{a}\right]+\left[\beta(p q)-A p^{a} q^{a}\right]
$$

for all $p, q \in[0,1]$. If we set $\gamma(p):=\beta(p)-A p^{a}, x:=p(1-q)$ and $y:=p q$, we see that (5.6.11) is equivalent to Cauchy's functional equation

$$
\gamma(x+y)=\gamma(x)+\gamma(y)
$$

for all $x, y \in[0,1]$ with $x+y \in[0,1]$. The continuous solution of (5.6.12) being $\gamma(x)=A^{\prime} x$ where $A^{\prime}$ is some constant. Thus

$$
\beta(p)=A p^{a}+A^{\prime} p
$$

on $[0,1]$. Direct verification shows that $\beta$ given by (5.6.13) indeed satisfies (5.6.9) if, and only if, $A^{\prime}=-A$. Thus from (5.6.6), (5.6.8) and (5.6.13) we get

$$
k(p, t)=p^{a} t+A p^{a}-A p
$$

for all $p \in[0,1], t \in I_{[0,1]}$. By using this explicit form of $k$ on $[0,1] \times I_{[0,1]}$, confining $v$ to $I_{[0,1]}$, and setting $q=0$ in (5.1.1), we get

$$
p^{a} k(1, u)+A p^{a}-A p=k(p, u)
$$

for all $p \in[0,1], u \in J$. This is the asserted (5.6.1) where $l(u):=k(1, u)$. A direct verification shows that a function $k$ defined by (5.6.1) satisfies (5.1.0) and (5.1.1) if, and only if, the constraints (5.6.2) are satisfied.

This proves Proposition 5.6.

Proposition 5.7. Let $k:[0,1] \times J \rightarrow R$ be a continuous map satisfying (5.1.0) and (5.1.1). Suppose there exists some $\left.\left.p_{0} \in\right] 0,1\right]$ such that the map $u \rightarrow k\left(p_{0}, u\right)$ is constant on $J$. Then $k$ is given by the following representation:

$$
k(p, u)=\operatorname{Act}(p, u)+p u_{0}-u_{0},
$$

where $u_{0} \in J$ is a constant and Act is a map satisfying the following properties:

(i) Act: $[0,1] \times J \rightarrow J$ is a continuous map,

(ii) Act $(p, \operatorname{Act}(q, u))=\operatorname{Act}(p q, u) \quad$ for all $p, q \in[0,1]$ and $u \in J$, 
(iii) Act $\left(p, u_{0}\right)=u_{0} \quad$ for all $p \in[0,1]$,

(iv) Act $(p, u)=u_{0} \quad$ for all $p \in\left[0, \frac{1}{2}\right], u \in J$.

Intuitively speaking, in view of (i) to (iii), the topological semigroup $[0,1]$ under usual multiplication acts on the topological space $J$ under Act, leaving a unique point $u_{0} \in J$ as a base point. Furthermore, in view of (iv), the space $J$ contracts to the "black hole" $u_{0}$ quickly.

The converse is also true.

Proof. Because of (5.1.1), the constancy of $u \rightarrow k(p, u)$ on $J$ implies the constancy of $u \rightarrow k(p q, u)$ on $J$ for all $q \in[0,1]$. Thus the set $\{p \mid u \rightarrow k(p, u)$ is constant on $J\}$ constitutes a non-degenerated subinterval of $[0,1]$ which contains 0 and $p_{0}$. This interval is also closed as $k$ is continuous, and so we can denote it by

$$
\begin{gathered}
{[0, b]=\{p \mid u \rightarrow k(p, u) \text { is constant on } J\},} \\
\text { where } b \in] 0,1] \text { is a constant } .
\end{gathered}
$$

We organize the proof into two steps.

STEP 1 . We shall prove that $b \geqq \frac{1}{2}$. For this purpose, we assume that $0<b<\frac{1}{2}$ and proceed to establish a contradiction.

Thus for each $q \in] b, 1-b[$, the maps $u \rightarrow k(1-q, u)$ and $v \rightarrow k(q, v)$ are non-constant on $J$, and we can apply Lemma 5.4 to equation (5.1.1) to conclude that there exist constants $\alpha(p, q), \beta_{1}(p, q), \beta_{2}(p, q)$ such that

$$
\begin{array}{ll}
k(p(1-q), u) & =\alpha(p, q) k(1-q, u)+\beta_{1}(p, q) \\
k(p q, v) & =\alpha(p, q) k(q, v)+\beta_{2}(p, q) \\
k(p, t) & =\alpha(p, q) t+\beta_{1}(p, q)+\beta_{2}(p, q)
\end{array}
$$

for all

$$
u, v \in J, p \in[0,1], q \in] b, 1-b\left[\text { and } t \in I_{q}:=\{k(1-q, u)+k(q, v) \mid u, v \in J\} .\right.
$$

Similarly to the proof of Proposition 5.6, the linearity of $t \rightarrow k(p, t)$ from the last equation in (5.7.4) can be extended to

$$
I_{[b, 1-b]}=\bigcup_{q \in[b, 1-b]} I_{q} .
$$

Thus there exist constants $\alpha(p):=\alpha(p, q), \beta(p):=\beta_{1}(p, q)+\beta_{2}(p, q)$ such that

$$
k(p, t)=\alpha(p) t+\beta(p)
$$

for all $p \in[0,1], t \in I_{[b, 1-b]}$. The second equation in (5.7.4) can be reduced to 


$$
k(p q, v)=\alpha(p) k(q, v)+\beta_{2}(p, q)
$$

for all $p \in[0,1], q \in] b, 1-b[, v \in J$. Based on (5.7.3) and (5.7.5), a contradiction will follow.

Let $q \in] b, 1-b[$ be arbitrarily chosen. The map $v \rightarrow k(q, v)$ appearing on the right hand side of (5.7.5) is non-constant on $J$, and thus

$$
\alpha(p)=0 \quad \text { iff } v \rightarrow k(p q, v) \text { is constant on } J .
$$

From (5.7.6) and (5.7.3) we get $\alpha(p)=0$ iff $p q \in[0, b]$ iff $p \in[0, b / q]$. The set of zeroes of $\alpha$ should be independent of the choice of $q \in] b, 1-b[$. This contradiction proves that $b \geqq \frac{1}{2}$.

STEP 2. For each $p \in[0, b]$, the map $u \rightarrow k(p, u)$ is constant on $J$, and we may define

$$
\varphi(p):=k(p, u)
$$

for all $p \in[0, b], u \in J$. It follows from (5.1.1) that

$$
\varphi(p)=\varphi(p(1-q))+\varphi(p q)
$$

for all $p \in[0, b]$ and $q \in[0,1]$. The continuity of $\varphi$ follows from that of $k$, and so the additive function $\varphi$ is given by

$$
\varphi(p)=p u_{0} \quad \text { for all } p \in[0, b],
$$

where $u_{0}$ is a constant. We now define Act: $[0,1] \times J \rightarrow R$ by

$$
\text { Act }(p, u):=k(p, u)-p u_{0}+u_{0}
$$

for all $p \in[0,1], u \in J$ to establish (5.7.1). It follows from (5.1.0), (5.7.7) and (5.7.8) that

$$
\text { Act } \begin{aligned}
(1-q, u) & =k(1-q, u)+q u_{0} \\
& =k(1-q, u)+\varphi(q) \\
& =k(1-q, u)+k(q, v) \in J
\end{aligned}
$$

for all $q \in\left[0, \frac{1}{2}\right], u, v \in J$. Hence, in particular, Act $\left(\frac{1}{2}, u\right)=k\left(\frac{1}{2}, u\right)+k\left(\frac{1}{2}, v\right)=\frac{1}{2} u_{0}$ $+\frac{1}{2} u_{0}=u_{0} \in J$. From (5.7.9) we also have

$$
\text { Act } \begin{aligned}
(1-q, u) & =(1-q) u_{0}-(1-q) u_{0}+u_{0} \\
& =u_{0} \in J
\end{aligned}
$$

for all $q \in\left[\frac{1}{2}, 1\right], u \in J$. This, together with (5.7.10), gives (i) and (iv) of (5.7.2). Putting $q=\frac{1}{2}$ into (5.1.1) we get (iii). Confining $q$ to $\left[\frac{1}{2}, 1\right]$ in (5.1.1) we get 
and so

$$
k(p, \operatorname{Act}(q, v))=p(1-q) u_{0}+k(p q, v),
$$

$$
(1-p) u_{0}+k(p, \text { Act }(q, v))=(1-p q) u_{0}+k(p q, v),
$$

which is equivalent to

$$
\text { Act }(p, \operatorname{Act}(q, v))=\operatorname{Act}(p q, v)
$$

for all $p \in[0,1]$. This proves (ii) in the case $q \in\left[\frac{1}{2}, 1\right]$. In the case $q \in\left[0, \frac{1}{2}\right]$, (ii) is implied by (iii) and (iv).

The converse can be directly verified. This completes our proof.

With the explicit forms of $k$ provided by Propositions 5.5 to 5.7, we now summarize the results in this section.

THEOREM 5.8. The regular canonical universal parallel composition laws PAR on an interval $J$ are given by either

$$
\operatorname{PAR}\left(p_{1}, p_{2}, \ldots, p_{n} ; u_{1}, u_{2}, \ldots, u_{n}\right)=\sum_{i=1}^{n} l\left(u_{i}\right)-(n-1) B,
$$

where $B$ is a constant and $l: J \rightarrow R$ is a non-constant continuous map satisfying the constraints (5.5.2); or

$$
\operatorname{PAR}\left(p_{1}, p_{2}, \ldots, p_{n} ; u_{1}, u_{2}, \ldots, u_{n}\right)=\sum_{i=1}^{n} p_{i}^{a} l\left(u_{i}\right)+A\left(\sum_{i=1}^{n} p_{i}^{a}-1\right)
$$

with the convention $0^{a}:=0$, where $a, A$ are constants with $a>0, a \neq 1$, and $l$ : $J \rightarrow R$ is a non-constant continuous map satisfying the constraints (5.6.2); or

(5.8.C) $\operatorname{PAR}\left(p_{1}, p_{2}, \ldots, p_{n} ; u_{1}, u_{2}, \ldots, u_{n}\right)=\sum_{i=1}^{n} p_{i} l\left(u_{i}\right)+C \sum_{i=1}^{n} p_{i} \log _{2} p_{i}$

with the convention $0 \log _{2} 0:=0$, where $C$ is a constant, and $l: J \rightarrow R$ is a nonconstant continuous map satisfying the constraints (5.6.4); or

$$
\operatorname{PAR}\left(p_{1}, p_{2}, \ldots, p_{n} ; u_{1}, u_{2}, \ldots, u_{n}\right)=\operatorname{Act}\left(p_{i 0}, u_{i_{0}}\right),
$$

where Act is a mapping satisfying (5.7.2), and $i_{0}$ is an index such that $p_{i_{0}} \geqq p_{i}$ for all $i \in\{1,2, \ldots, n\}$.

\section{Examples of measures and their composition laws.}

The subsequent discussions are all informal.

EXAMPLE 6.A. If a procedure $\mathscr{A}$, written in a certain programming language, consists of $n$ internal procedures $\mathscr{A}_{1}, \mathscr{A}_{2}, \ldots, \mathscr{A}_{n}$ which are mutually disjoint, then the static statement count of $\mathscr{A}$ is the sum of those of the $\mathscr{A}_{i}$ 's. Thus the 
static count of number of programming statements in a procedure is governed by the composition law (5.8.A) with $l: R \rightarrow R$ the identity map and $B=0$.

If the probability distribution $\left(p_{1}, p_{2}, \ldots, p_{n}\right)$ measures the relative frequencies of the number of calls to the $\mathscr{A}_{i}$ 's during execution, then in this measure $\left(p_{1}, p_{2}, \ldots, p_{n}\right)$ has no direct bearing on the static statement count of $\mathscr{A}$. It is not a surprise that its composition law (5.8.A) is independent of the $p_{i}$ 's.

Example 6B. Let $\Gamma$ be the space of all finite complete probability distributions. If $Q_{1}, Q_{2}, \ldots, Q_{n},\left(p_{1}, p_{2}, \ldots, p_{n}\right)$ are in $\Gamma$, we can form a distribution in $\Gamma$ by

$$
\sum_{i=1}^{n}\left(p_{i}, Q_{i}\right):=\left(p_{1} q_{11}, p_{1} q_{12}, \ldots, p_{1} q_{1 k_{1}} ; \ldots ; p_{n} q_{n 1}, p_{n} q_{n 2}, \ldots, p_{n} q_{n k_{n}}\right),
$$

where $Q_{i}=\left(q_{i j}\right)_{j=1}^{k_{i}}$.

If we initialize a measure $\mu$ on $\Gamma$ by $\mu(1)=0$ where (1) is the trivial distribution of the sure event, then, under the composition law (5.8.B) with $l: R \rightarrow R$ taken to be the identity map, the measure $\mu$ so induced on $\Gamma$ is explicitly given by

$$
\mu\left(p_{1}, p_{2}, \ldots, p_{n}\right)=A\left(\sum_{i=1}^{n} p_{i}^{a}-1\right)
$$

for all $\left(p_{1}, p_{2}, \ldots, p_{n}\right) \in \Gamma$, since

$$
\begin{aligned}
\mu\left(p_{1}, p_{2}, \ldots, p_{n}\right) & =\mu\left(\sum_{i=1}^{n}\left(p_{i},(1)\right)\right) \\
& =\sum_{i=1}^{n} p_{i}^{a} \mu(1)+A\left(\sum_{i=1}^{n} p_{i}^{a}-1\right)=A\left(\sum_{i=1}^{n} p_{i}^{a}-1\right) .
\end{aligned}
$$

This measure has been introduced and studied in [2] and [3].

If we initialize a measure $\eta$ on $\Gamma$ by $\eta(1)=0$ as before, and assume that $\eta$, in exponential scale, has the composition law (5.8.B) with $l: R \rightarrow R$ taken to be the identity map, and with $A=0$, then the measure $\eta$ so induced is given by

$$
\eta\left(p_{1}, p_{2}, \ldots, p_{n}\right)=\log _{b}\left(\sum_{i=1}^{n} p_{i}^{a}\right)
$$

for all $\left(p_{1}, p_{2}, \ldots, p_{n}\right) \in \Gamma$. This follows from

$$
\begin{aligned}
\eta\left(p_{1}, p_{2}, \ldots, p_{n}\right) & =\eta\left(\sum_{i=1}^{n}\left(p_{i},(1)\right)\right) \\
& =\log _{b}\left(\sum_{i=1}^{n} p_{i}^{a} b^{\eta(1)}\right)=\log _{b}\left(\sum_{i=1}^{n} p_{i}^{a}\right) .
\end{aligned}
$$


When the positive constant $b \neq 1$ is chosen to be $2^{1-a}, \eta$ is Renyi's entropies of order $a$ (cf. [8]).

ExAmPLE 6.C. Similar to Example 6.B, the entropies introduced by Shannon ([9])

$$
H\left(p_{1}, p_{2}, \ldots, p_{n}\right)=C \sum_{i=1}^{n} p_{i} \log _{2} p_{i}
$$

on $\Gamma$ has the initialization $H(1)=0$, and the composition law (5.8.C) with $l$ : $\mathbf{R} \rightarrow \mathbf{R}$ the identity map.

Any measure $\mu \mathscr{A}$ which is the mathematical expectation of the measures $\mu \mathscr{A}_{i}$ of its modules is generated under the composition law (5.8.C) with $C=0$.

EXAMPLE 6.D. Intuitively speaking, a measure $\mu \mathscr{A}$ which is governed by the composition law (5.8.D) depends only on the measure $\mu \mathscr{A}_{i 0}$ and the probability $p_{i_{0}}$ of its most likely component $\mathscr{A}_{i_{0}}$.

An example of an action on an interval $J$ satisfying (5.7.2) is the following flow:

$J$ : a finite interval of $R$ with end points $e_{1}<e_{2}$.

Act: $[0,1] \times J \rightarrow J$ defined by

$$
\text { Act }(p, u)= \begin{cases}\min \left\{u+\left(e_{1}-e_{2}\right) \log _{2} p, e_{2}\right\} & \text { if } p>0 \\ e_{2} & \text { if } p=0\end{cases}
$$

Extensive literatures on various measurements of information has been summarized by Aczél [1]. The general structure of Act has been studied by Stadtlander [10]. A general discussion on the axiomatic foundation of measures of information can be found in the paper of Forte [4]. There is connection between (5.6.9), in the case $a=1$, and the information function studied by Tverberg [11].

The author is grateful to the referee for his many useful remarks.

\section{REFERENCES}

1. J. Aczél and Z. Daróczy, On measures of information and their Characterizations, (Mathematics in Science and Engineering, Vol. 115), Academic Press, New York -San Francisco - London, 1975.

2. N. L. Aggarwal, Y. Cesari and C. F. Picard, Propriétés de branchement lieés aux questionnaires de Campbell et à l'information de Renyi, C. R. Acad. Sci. Paris Sér. A 275 (1972), 437-440. 
3. F. Charvát and J. Havrda, Quantification method of classification processes, Kibernetika Cislo 1, Rócnik 3 (1967), 30-35.

4. B. Forte, Measures of information: the general axiomatic theory, Rev. Française Automat. Informat. Recherche Opérationelle Sér. Rouge, $3^{\mathrm{e}}$ annee, R-2 (1969), 63-90.

5. C. T. $\mathrm{Ng}$, On the functional equation $f(x)+\sum_{i=1}^{n} g_{i}\left(y_{i}\right)=h\left(T\left(x, y_{1}, y_{2}, \ldots, y_{n}\right)\right)$, Ann. Polon. Math. 27 (1973), 329-336.

6. C. $\mathrm{T} . \mathrm{Ng}$, Representation for measures of information with the branching property, Information and Control 25 (1974), 45-56.

7. C. $\mathrm{T} . \mathrm{Ng}$, On the measurable solutions of the functional equation

$$
\sum_{i=1}^{2} \sum_{j=1}^{3} F_{i, j}\left(p_{i} q_{j}\right)=\sum_{i=1}^{2} G_{i}\left(p_{i}\right)+\sum_{j=1}^{3} H_{j}\left(q_{j}\right),
$$

Acta Math. Acad. Sci. Hungar. 25 (1974), 249-254.

8. A. Renyi, On measures of entropy and information -I, 4th Berkeley Symp. Math. Statist. and Probability (1960), 547-560 (University of California Press, Berkeley).

9. C. E. Shannon, A mathematical theory of communication, Bell System Tech. J. 27 (1948), 379 423.

10. D. Stadtlander, Thread Actions, Duke Math. J. 35 (1968), 483-490.

11. H. Tverberg, A new derivation of the information function, Math. Scand. 6 (1958), 279-298.

UNIVERSITY OF WATERLOO

FACULTY OF MATHEMATICS

WATERLOO, ONTARIO N2L 3G1

CANADA 